\title{
SCATEAgent: Context-Aware Software Agents for Multi-Modal Travel
}

\author{
Min Yin and Martin Griss \\ Computer Science Department \\ University of California at Santa Cruz \\ myin@cse.ucsc.edu,griss@soe.ucsc.edu \\ (To appear, AAMAS Workshop on Agents in Traffic and Transportation, July 2004)
}

\begin{abstract}
In this paper we describe our research on an agentbased intelligent, flexible, and context-aware multimodal traveler information system, SCATEAgent. The work targets the representation and manipulation of core user, preference, and context models which facilitate highly-customized and adaptable agents playing key roles in an agent-based Advanced Traveler Information System (ATIS). This ATIS finds and fuses information from multiple sources on routes, congestion, incidents, weather, alternative transit modes and schedules, provides proactive real-time updates and context-specific guidance as the user travels, and is vigilant about impacting events. A highly intelligent, personalized, and user-friendly assistant to the traveler, especially in case of transitions among different travel modes, SCATEAgent will promote effective individual traveling and also help to smooth the transportation load in general. The expected contributions include the design of user, preference, context and travel ontologies; user, context, task models based on these ontologies, a set of representations to drive agent behavior and communication, and a compatible integration of rules, machine learning, information retrieval, and semantic web. Currently, we have completed the first stage of our research, producing first pass ontologies and models, an initial prototype of a small-scale test-bed incorporating GPS-enabled cell-phones, and multiple mechanisms for agents to handle and adapt to such models and travel related events.
\end{abstract}

Keywords: Software Agent, Travel, Contextawareness, Modeling, ATIS

\section{Introduction}

Travelers using their personal or shared vehicles, or public transit options, are facing increasing congestion, more incidents, and longer delays. Most surface travel is done in single occupant vehicles (SOV), on primary highways and some arterial roads. Most of the driving is done by commuters on their regular routes, who often spend over $50 \%$ of their time delayed by traffic [Telcontar]. Too few drivers consider alternate routes, adjust schedules, or select alternate modes of transit. Effective Transportation Systems Management is critical for supporting the nation's continued population and economic growth. The evolution of Intelligent Transportation Systems has shown that effective solutions must combine both supply-side traffic management techniques to control traffic flow and demand-side initiatives to encourage more efficient travel choice behavior. The challenge is to find and implement solutions that achieve an efficient reallocation of network capacity over time and space without seriously violating any individual user's preferences for mode, routing, departure, and/or arrival time. Additional value would be to recommend ancillary services appropriate to the user's priorities, location and needs.

Our work focuses on the demand side, seeking to improve the usability and quality of the advice given by an Advanced Traveler Information System (ATIS). In a Department of Transportation study and analysis of a survey of ATIS needs and customer types [Lapin 2000; Lapin 2000a], Lapin identifies several types of ATIS customers and their differing needs. She notes distinct gender related preferences, different levels of comfort with technology, and interest in differing kinds of ATIS advice. This analysis highlights the opportunity for a more dynamic, flexible ATIS that adapts its behavior to the specific user's needs, preferences, and activity at the time of planning and especially while executing a trip. For Mobile ATIS Lapin states "Drivers want reliable, accurate, relevant traffic information while driving. For many trips, the traffic information provided pre-trip is outdated when the driver reaches a potential route diversion decision. This is where the greatest demand for ATIS exists. Drivers recognize the safety challenge of delivering information to a driver, and most respondents expressed safety concerns about mobile phone use when driving. Nevertheless, drivers wish they could press an ATIS button when approaching congestion or a route choice and quickly know which route would be least congested." 
Cell-phones are a primary potential tool for interacting with an ATIS, particularly if the interaction could be highly personalized, mostly hands-free, and very context-specific in order to reduce distraction and danger. In 2000, there were over 120 million cell phones in use in the United States, at least 500,000 drivers were using cell phones at any given time during the day, and more than $50 \%$ of all drivers had wireless phones in their vehicle at all times [Cellphone]. Tomorrow's ubiquitous computing environment will be filled with even more intelligent, location-aware devices: on your person, in your office, car, bus, train and home, on the street, in stores, movies, museums and restaurants. Future devices and systems will exhibit emergent intelligence - the seeming ability to act intelligently by integrating a variety of information and resources pertinent to the user's current situation.

Nowadays agent technologies have seen a lot of use to support mobile users [Griss et al., 2002, Goni, et al, 2001, Blechschmitt \& Strodecke, 2002]. The autonomy, intelligence and collaborative nature of agents play a key role in mobile systems and applications, such as personal email systems, personal news systems, wireless web applications, etc. The key idea is to apply software agent technology to support these mobile users. Intelligent agent technology promises to provide new levels of highly personalized, adaptive, context-aware functionality. The combination of wireless mobile appliances and intelligent agents using physical context to mediate the interaction with many services would greatly enhance the user's ability to plan, select and execute travel, and to choose appropriate travel modalities, routes, schedules, and relevant ancillary services.

SCATEAgent is a set of agents, utilities, and components developed to implement a portion of this vision. It leverages agent technology to provide personalized, dynamic and context-aware services to the mobile traveler. Our key contributions are in the areas of the specific ontologies and models we have designed to represent trips, services, and traveler preferences to enable intelligent multi-modal travel support. We have also novel approaches to the architecture of the agentbased ATIS and to the way we have integrated the rules and the agents.

The rest of the paper is organized as follows. Section 2 gives a brief description of the architecture of SCATEAgent and the integrated agent-based information processing system. Section 3 describes the ontologies and models, Section 4 introduces related technologies. Section 5 describes our current working status, briefly introduces the SCATEAgent test bed and summarizes plans for evaluation of the system. Finally, Section 6 concludes with a discussion of future work.

\section{System Architecture}




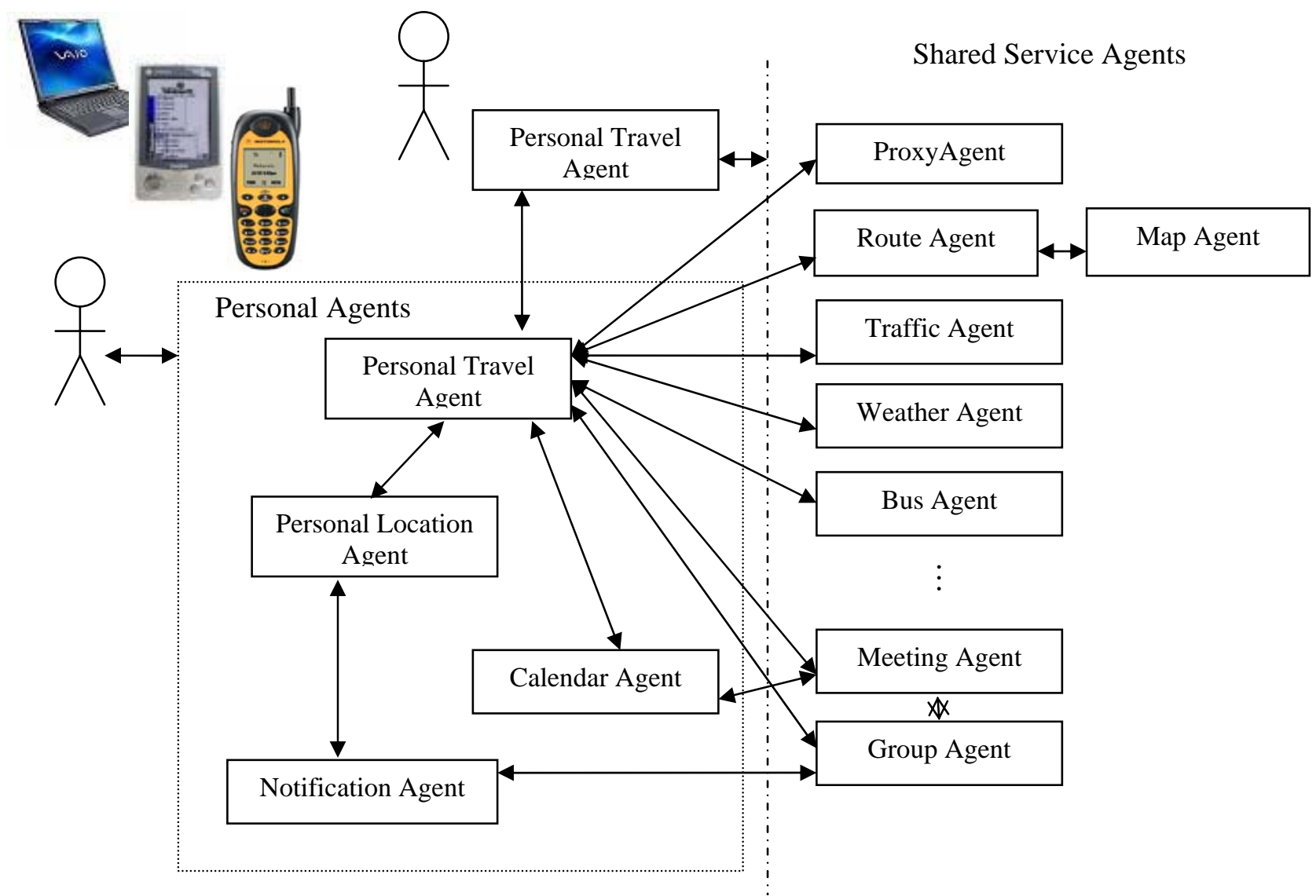

Figure 1. SCATEAgent System Architecture

Figure 1 shows the SCATEAgent system architecture. There are two sets of agents, personal agents and shared public service agents. The personal agents include Personal Travel Agent (PTA), Personal Location Agent (PLA), and Calendar Agent. These agents belong to each individual. They maintain and manage the personal preferences and personal activities. For example, PTA is responsible for individual trip planning and monitoring and it utilizes and manages all travel related personal preferences such as preferred transportation types, route chosen history, special travel requirements due to disability or other medical situations, etc. PLA works closely with PTA. It keeps track of the traveler's location and reports it constantly to the PTA so that PTA is able to monitor the trip and propose prompt, accurate and relevant information to the traveler or prepare to re-plan the trip when it detects unexpected situation such as delay. PLA maintains location related personal preferences such as points of interests (POI), higher-level models of location ("at home", "at work", etc.), location history, location security, and location authorization information. The location information provided by the PLA can also be used by other personal agents. For example, notification agent can consult PLA and choose appropriate notification channel according to the user's current location. On the other hand, before the calendar agent inserts an event entry in its schedule, it may check with PTA to make sure that the location of the proposed event is accessible by the personal at the proposed time slot.

For PTA to plan the trip accurately and precisely, it also needs to get information, such as maps, weather, traffic, and public transportation schedule from external services. These services are provided by shared public service agents. One great advantage of using agent technology comes from its loosely coupled architecture. For example, there can be multiple map agents; each provides map service from different source or with different granularity. This way, we can prioritize them according to factors such as reliability, cost, promptness, etc. If one service is not available for some reason, the agent architecture will automatically find the next service agent which provides the same service using a facilitator agent. This is also true for the route agents, which provide routing services to the PTA. There might 
be different route agents, some are simple, fast, but less accurate; some are more precise and sophisticated, but not free. PTA may choose to use one of the route agents, or it may chose to consult more than one and then select a route among those returned by different route agents. Such decisions are made according to traveler's concerns, preferences, and the context of the trip.

The PTA plans trip in a goal-directed and task-driven manner. For example, when the Meeting Agent has planned a meeting that requires the physical attendance of all or part of the participants, it will send a task, or a travel request, to those participants. Upon the recipient of the request, the PTA will then check the person's current location with the PLA. Obviously there will be no need to plan a trip if the meeting will be held in the same building. But if moderate travel is required, the PTA will react and function accordingly. Similarly, a task can be sent to a group of people through the Group Agent when all the individuals in such group are supposed to travel to the same destination.

Finally, the interaction between human and agents is done through mobile or stationary devices such as cell phones, PDAs, laptops, or even a public kiosk. It is important to have a Proxy Agent to monitor and manage such human to agent interaction. Actually, since the traveler is always on the move, it is important to have a uniform human-agent interface and a set of interaction agents to maintain a continuous seamless dialogue between the user and different devices.

In the architecture presented in Figure 1, it is essential to develop a set of ontologies understood and agreed by all agents. The user preferences, context, and tasks will be represented by corresponding models based on those ontologies. More details will be given in the following sections.

\section{Ontologies and Models}

In SCATEAgent, we need a coherent and effective way of creating/maintaining/ensuring consistency, and using several preference, information and context models to drive complex decisions.

Generally, agents communicate through asynchronous message passing, using a looselyformatted, text-based communication language.
Messages include a header (to, from, etc.), an ontology, a message type, an expected message protocol, and a variable-length body. FIPA specifies a dozen or so highlevel message types, such as Notify, Query, Inform, etc. In addition to requests to perform, communication languages allow agents to exchange information such as commitments, goals, beliefs, and intentions.

Different agents (Weather Agent, Bus Agent, Traffic Agent, etc) of the SCATEAgent test-bed need to access a variety of information and services, such as maps, schedules, incidents, and calendars from multiple sources, with heterogeneous interfaces. Some are proprietary applications (e.g., OutLook, Palm desktop), while others are databases, FTP accessible services (e.g., PeMS), HTTP accessible web-sites and recently some WSDL described web services (e.g. Microsoft MapPoint). This means that shared service agents, or service wrappers will need to convert these idiosyncratic interfaces into a standardized agent-compatible format and harmonize two different view points on ontology and knowledge representation: the web services view uses XML, RDF, semantic web (JENA) and WSDL languages, while the $\mathrm{AI} /$ agents style uses the more logic oriented KIF, KQML/ACL, and DAML/OIL approaches.

A different approach is to totally discard standard structured agent languages such as FIPA-SL and promote relatively weak ontologies through a Semantic Web standard such as RDF [Grimnes, et al.]. The advantages of such approach are that it costs very little to integrate existing web services into the agent system and it is relatively easy to reuse weak ontologies elsewhere when necessary. But we suspect it will complicate the agent negotiation and learning abilities.

We believe for our SCATEAgent system, the solution lies between the two extremes. Ideal would be ontologies and models that leverage both sides appropriately and are tailored to meet the specific ATIS needs. We are not able to find such ontologies and models in the literature. Other desirable features include ensuring agent autonomy, providing context-awareness, controlling the flow of ACL messages between agents, and enhancing flexibility and scalability.

Figure 2 gives a fragment of the travel ontologies and models used in SCATEAgent. The models are described as objects, object attributes and object relations. 


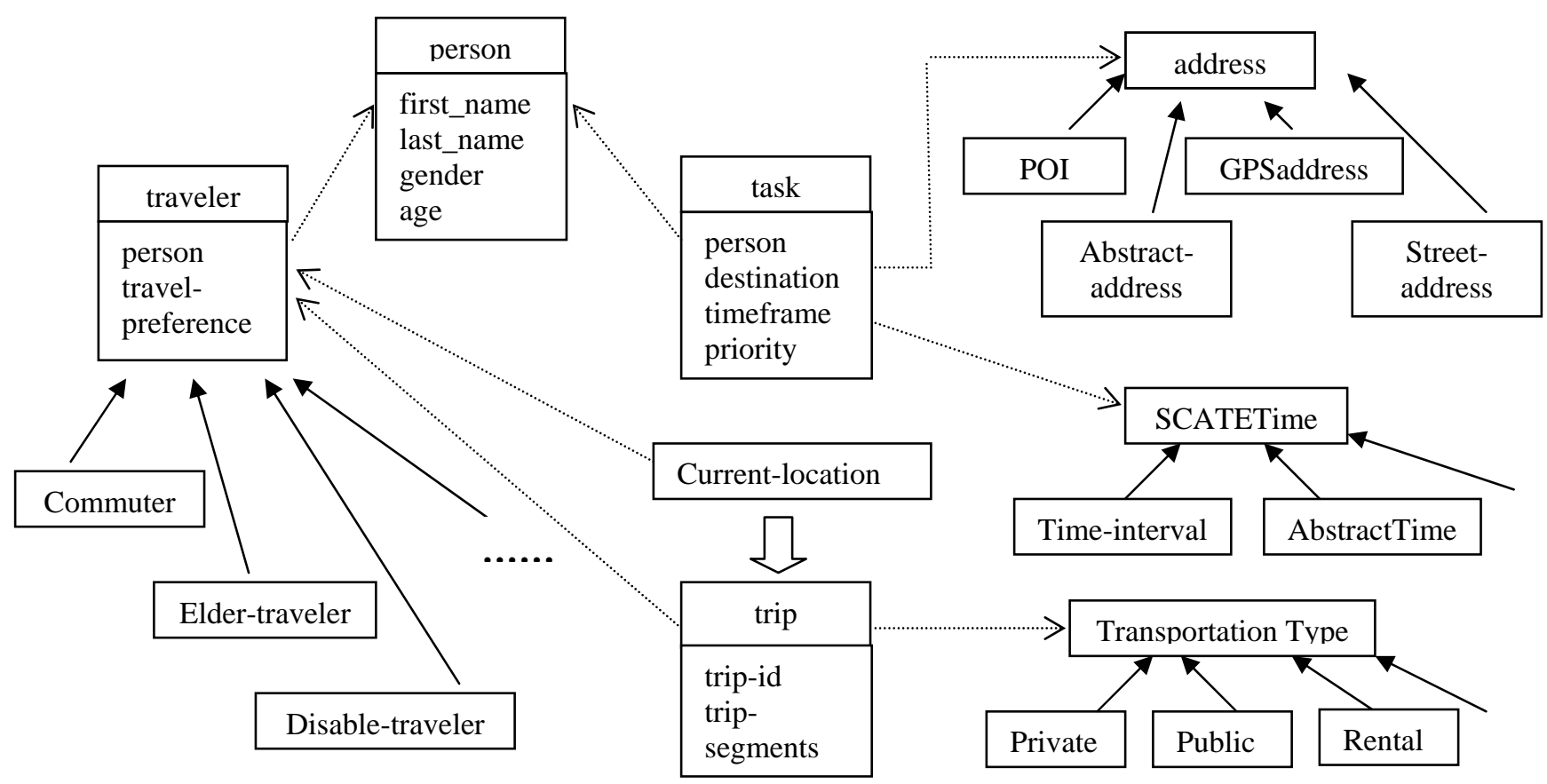

Figure 2 Ontologies and Models Example

In Figure 2, the solid arrows represent inheritance between objects while the dashed arrows represent the reference relationships. For example, "POI" and "abstract-address" are two different kinds of address. "POI" is the personalized points of interest such as home, office, school, etc, while "abstract-address" represents one special place such as "coffee”, "bus stop”, etc. Traveler model refers to person model for the general information of the traveler. Commuters, eldertraveler, and disabled-travelers are all different kinds of travelers; each having their own set of characteristics. The wide arrow indicates that a trip plan can be generated according to a travel task, the traveler's current location, the traveler's personal preference, and available transportation tools.

SCATEAgent is constructed using the JADE platform with the JESS rule engine [Jess]. Currently the models are represented with a combination of JESS templates, facts and Java objects. There are several advantages of using JESS facts as ontology instances. First, agents can easily adjust their behaviors and demonstrate intelligence and flexibility by directly applying JESS rules to the elements of the user model and context models. Adding, deleting, or modifying JESS facts and rules is of lower cost as compared with changing the states of a state machine so both the models and the rules can evolve gradually with increasing complexity. Second, a JESS fact can be easily wrapped with an ACL message and be sent among the agents. Finally, JESS facts and Java objects can also be converted conveniently into each other so that SCATEAgent can exchange data with external services, especially web services. For example, a "trip" fact and the "trip-segment" facts it refers to can be converted into a java object of the "scate.travel.Trip" class, which is in turn compatible with other data formats, such as the SOAP route object provided by Microsoft MapPoint map and routing services, through intermediate utilities.

Figure 3 gives a very simple example of how a trip is planned according to some given facts. Figure 3 (a) is some traveler related facts, including traveler, address, transportation type and task. For the sake of simplicity, the time slots are omitted. Figure 3 (b) gives a simple trip planning rule using the facts given in (a). It claims that the traveler can travel by a rental car if his/her personal car is not available, he/she does not exclude rental car from the candidate transportation means, the trip budget is "mid" or "high", and both the source and the destination can be reached by car. Figure 3 (c) gives the class structure of the Java object compatible to the fact generated by the rule in Figure 3 (b). Notice that rule in Figure 3 (c) only chooses a possible transportation tools without specifying the route details. Such routing task will be then delegated to Route Agent, such as MapPoint Route Agent. Figure 3 (a) and Figure 3 (c) show how ontologies are expressed as JESS facts, JESS fact slots, and Java class fields. Other approaches, such as Protégé, are also being explored so that the ontologies developed can be generated compatibly to DAML/OIL or OWL, yet still enacted using Jess. 


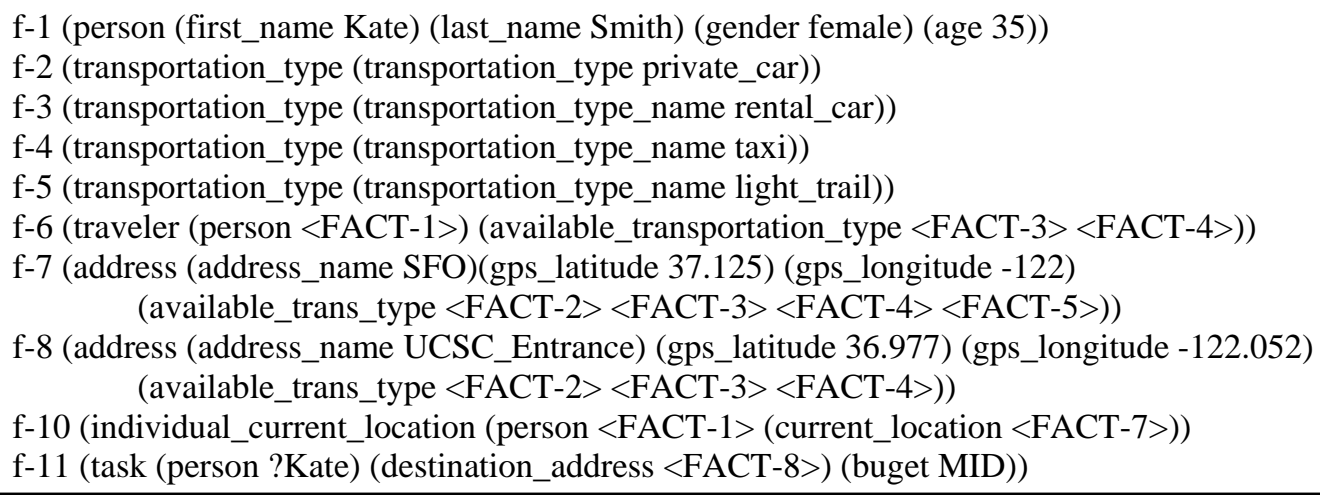

(a) Some Travel Related Facts

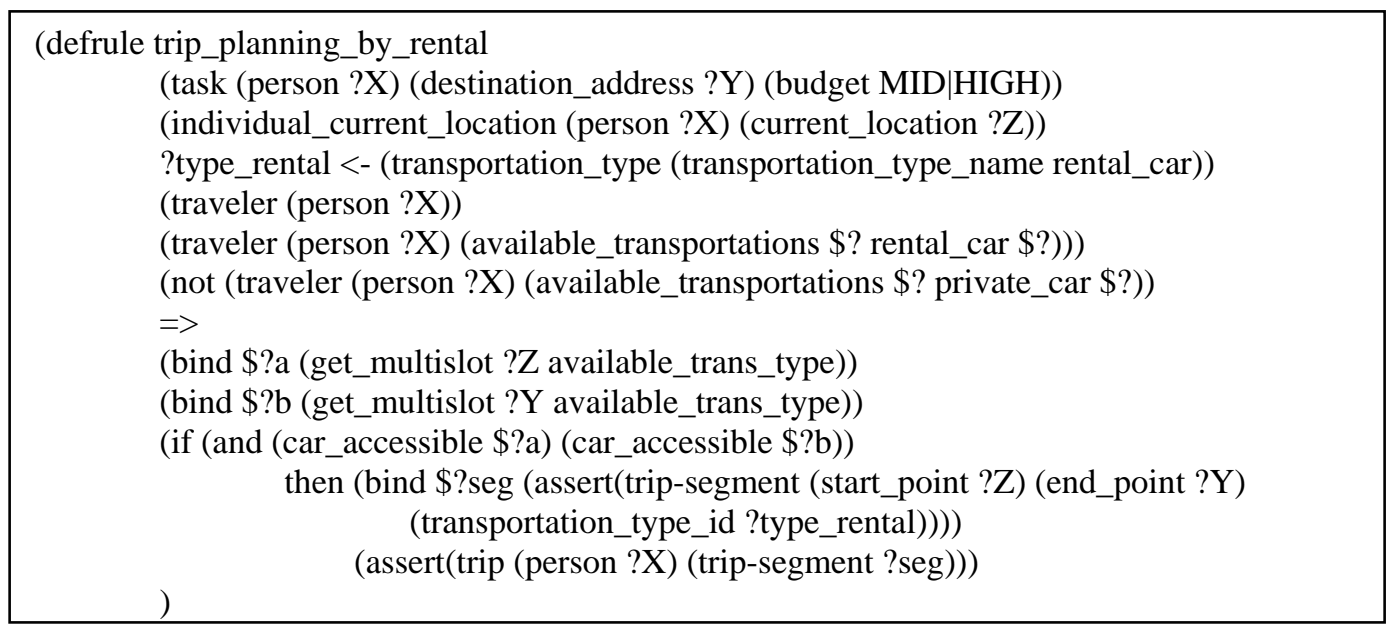

(b) A Simple Trip Planning Rule

\begin{tabular}{|ll|}
\hline Class trip \{ & Class RouteSegment \{ \\
& Address end_point; \\
Traveler traveler; & TransType type; \\
SCATETime start_time; & Double length; \\
Address start_location; & SCATETime end_time; \\
ArrayList segments; //array of RouteSegment & \\
\hline
\end{tabular}

(c) Corresponding Java classes

It can be seen that both the context (travel budget, personal car availability) and the user preferences (excluded transportation means) play an important role in the selection of transportation means. It is also relatively easy to add, delete, or modify the rules, for example, to change the budget threshold from "mid or high" to "high", etc. One or more possible routes might be returned by one or more Route Agents. SCATEAgent will then query the Traffic Agent and Weather Agent for the traffic and weather situation of the interested route segment. The results will be transferred as JESS facts and trigger relevant rules to select the best route for the current traveler under the current travel context.

The process of choosing public transportation means, including public transits, is relatively easy. Most public transportation schedules are pre-stored in the knowledge base as facts and are updated on a regular base. A route can be generated by applying rules that matches both the transit address and the transit time of different public transportation schedules.

Once an initial route is generated and the traveler is on his/her way, the PTA monitors the trip using the updated location information reported by the PLA. Each new location insert a new fact into the knowledge base and any possible delay or change of the plan will be caught by corresponding rules which will consequently trigger the process of notifying the user and generating new travel plans according to the unexpected situation.

Besides the primary travel task, other smaller tasks or tasks of lower priority can come into play via adding 
relevant rules. For example, tasks listed in the personal to-do list, such as pick up laundry or visit the dentist, can affect the travel route, the time, and the transportation means.

\section{Related Work and Technologies}

There is considerable relevant work in the areas of software agent technology, distributed AI, and contextaware computing, applications of agent technology to traffic management systems. We will only mention a few of the most important to our work. Many of those efforts provide a base for our research, but are not directly comparable because we focus on an individually usable and personalized ATIS, while other agent-based traffic applications focus on simulation or traffic management.

Intelligent multi-agent systems are seeing increasing use in research and products associated with complex adaptive systems. Agent technology has matured considerably via standards by the Foundation for Intelligent Physical Agents (www.fipa.org), open source agent toolkits, such as JADE [Jade], large-scale agent interoperability tests (www.agentcities.org) and numerous applications of agents in research, advanced development and products [Griss, 2000]. Agents have been applied to enterprise integration and supply chain management, planning, scheduling and execution control, materials handling, and inventory management. In our work, we build on the JADE system, building on a prior integration with the JBOSS J2EE server [BlueJADE]), developed to increase large-scale agent scalability and manageability.[Griss \& Kessler, 2002].

\section{Applications of Agents to Traffic}

Multi-agent systems are well-suited for use in intelligent transportation systems. [Burmeister et al, 1997] states that agents can work well in complex systems in which:

- the problem domain is geographically distributed,

- $\quad$ subsystems exist in a dynamic environment, and

- subsystems need to be adaptive and interact more flexibly.

According to Adler and Blue, efforts to provide assistance to drivers using information technology began in the 1950's [Adler 1998]. These early systems involved both traffic surveillance and real-time dissemination of advisory notices, primarily through the use of visual displays. The development of in-vehicle route guidance systems (IVRGS) began in the 1970's [Rosen 1970]. IVRGSs, including GPS-enabled cellphones, have evolved to include real-time updates of traffic incidents and congestion. In the last few years, the US Department of Transportation has developed a
National Intelligent Transportation System Architecture designed to provide "a common framework for planning, defining, and integrating intelligent transportation systems."[NITSA]

Today, large amounts of data on traffic, weather, dynamically annotated maps, and driving directions are made available on the Internet by Caltrains, the Department of Transport, NOAA, and many others. Research groups and product companies are collecting and consolidating this information and using it to offer real-time advice to travelers, either during trip planning or en route [FreeWay2000, PeMS, TrafficDodger, Telcontar, WestwoodOne]. Real-time updates can be sent via web, email, pager, cell-phone, or other wireless technology [MobilityTechnologies, SFBayTraffic]. Pretrip planning can include maps showing incidents and current traffic flows or an annotated "best-route" with estimated travel time based on current congestion and incident information [TrafficDodger]. Some also offer a degree of personalization [Iteris, SFBayTraffic]. In addition to GPS-enabled in-vehicle systems, laptops, PDA's and cell-phones can now offer maps and turn-byturn directions [ViaMoto, TeleNav]. Motorola's ViaMoto service uses GPS-enabled i88s phones to offer location-aware and navigation services to ATIS and fleet management providers. GPS-equipped public transit vehicles can send notifications to web-pages, a user's cell-phone, or PDA when the next bus is due to arrive [CoolTown, Kindberg 2002, NextBus]. All these services provide a solid foundation for SCATEAgent. Currently we have a bus agent and two route agents, more service agents will be developed as the system evolves.

Distributed agents have been used in a variety of surface and air traffic simulation and management systems [Andersson \& Rnnbom 1999; Appiani 1999, Erol 1999, Fernandes \& Olivera 1999, Hidas 2002, Katwijk 2002, Roozemond 1996, Wojcik 2000]. Compared to traditional micro-simulators such as PARAMICS, distributed agent-based simulators offer increased flexibility, the ability to model driver behavior and near real-time simulation and prediction. Agents, neural networks, and other AI techniques have been applied to incident analysis, and to simulate features and usability of an ATIS [Das 1999]. CityTraffic combines real-time sensors with a high-performance distributed agent system for real-time prediction [CityTraffic]. Intelligent Automation, Inc. has used agents for several real-world traffic simulators and controllers, allowing some experimentation with driver behavior [IAI].

The Oct-Dec 2002 issue of Transportation Research Part $\mathrm{C}$ was devoted to the growing use of agents as an emerging technology for transportation. While most of the papers were on simulation or ATMS coordination and analysis, Adler and Blue [Adler 2002] described a 
cooperative multi-agent transportation management and route guidance system linking ATMS and some ATIS capabilities. In an earlier paper they make a compelling case for the role of AI techniques in "3rd generation Intelligent Traveler Information Systems,” highlighting the need for a more personalized and adaptive ATIS [Adler 1998]. They stress the key role of machine learning and other AI techniques in delivering a more pleasant and effective experience to travelers while planning a trip or en route; however, they make no mention at all of agents as a vehicle to deliver the needed capability in a dynamic, incremental and distributed fashion.

\section{Context- and Location-Aware Systems}

There are numerous approaches through GPS, wireless, RFID, infra-red and Bluetooth, and other peerto-peer and ubiquitous computing technology to determining the physical location of appliances, and the availability and presence of other appliances and local services in the neighborhood [CoolTown].

Future cell phones will do much more than make calls and keep phone books. Perhaps the greatest potential that they hold will require sensing the environment, interacting with nearby devices, and exhibiting opportunistic behavior specific to current user goals and context.

The basic idea of seeking and discovering the ability of neighboring devices or services is similar to that of SUN's peer-to-peer Jini [Jini] or UUDI/XML-based W3C web services. In Jini the advertising of abilities uses an object's API of public method interfaces, while in web-services a UDDI registry contains a higher-level WSDL description in XML.

Currently we are using the GPS-enabled cell phones to determine the traveler's location. Future work will introduce other location-aware devices, most probably infra-red and Bluetooth, and use rule-based goals and abilities to enable the matching and negotiation to occur in a much richer and more semantically meaningful way.

\section{Agent Intelligence and Coordination}

Some agent toolkits provide some useful rule-based and goal-based capabilities. For example, the Zeus agent toolkit [Zeus] has a built in problem-solver, planner, and rule-based goal/fact system, as well as a protocol-based negotiation system. Rules and workflows can be used to define how agents with different roles interact in a flexible, yet constrained manner to accomplish a collaborative task [Griss 2000; Chen et. al., 2000, Sutton \& Osterweil, 1997]. Others have used UML state machine and interaction diagrams, and (colored) Petrinets to provide more precise specification and control of agent interactions. In our work, we are integrating rule- based and state-machine based approaches, where the rules provide flexible handling of transitions in the state machines, and the state machines provide structured context for the rules and goals.

There is significant work on the development, extension and application of UML-based modeling for agent systems, AUML [Odell, 2001; Bauer, 2001], and also the development and evaluation of complete agent system development methods, such as AOP [Shoham, 1993], GAIA [Wooldridge et. al, 2000], and Tropos [Guinchiglia, et al, 2003]. One of the MIT Oxygen projects proposes to formalize goals as a language construct - an interesting idea, but little has yet been published [Ward et al, 2002]. The development of agent society patterns in UML [Kendall, 1999], has implications for analysis and design, and leads to aspectoriented implementation.

\section{Status and Evaluation}

We are in the process of constructing and revising a small multi-agent ATIS test-bed. This experimental platform will be used to tune and evaluate the improved models and rule-based technology. We have completed the first stage of constructing models and ontologies and built our test-bed to run the first prototype of SCATEAgent. The system uses the JADE agent framework and JESS rule system. Currently, it uses several Java-based GPS cell-phones (Motorola i58sr), interacting with personal assistants, location and notification agents, which access local bus schedules, maps, routing services, and traffic information in the Santa Cruz area. One of the more challenging tasks was interfacing the phone with the agents. The J2ME program running on the phone will periodically poll an IP socket exposed by a specific proxy phone agent to exchange encoded messages and GPS location with other agents. Agents representing drivers and travel information services will help travelers plan a route and drive it or take public transport. The personal agents interact with other agents, with calendars and emails, and use VoiceXML,CallXML, or the phone proxy to notify the phones.

The current test-bed PTA, is customized via a simple profile (name, home and office address, phone and cell phone numbers) and some simple preference rules ("I prefer to take the bus, unless I have an urgent meeting later"; "I am willing to delay my trip to avoid traffic.”). We are in the process of integrating the richer models described in this paper into the system.

Currently SCATEAgent runs under the following small test scenario:

- Start at work, and issue the command "Go home". 
- A transportation method, car or bus, will be selected by PTA according to the user's preference and it will be proposed to the user.

- Upon the confirmation of the user, the PTA will select the appropriate driving route or bus route.

- The notification agent will notify the user to leave when the target bus is approaching.

- The PLA will monitor the trip, notify of the next transit or possible bus delay

A future target test scenario incorporating results of the proposed research is something like:

- Start at work, and issue the command "Go home".

- Some users will get advice on which bus to take, from which stop, and when. Others will be reminded where they parked their car.

- Directions will be offered for the recommended route, adjusting for anticipated traffic at that time.

- Some users will be reminded that they should pick up dinner before coming home, or that they should pick up the kids at ballet, and the route and directions adjusted for that activity.

- $\quad$ The agents will display some vigilance related to congestion, incidents, or changes in schedule or plans en route. ("e.g., the stop lights at Mission are out, take the alternate route; or location of meeting with friend just changed").

The results of the first scenario test are promising and we are enhancing the system gradually. An initial evaluation will be done by the small group of researchers using a test harness for the test-bed. Simulation components will feed in events to simulate new routes, new personal goals or preferences, load and incidents, and weather. As the system becomes tuned and new components are added, we will incrementally replace the simulated components, and have the researchers or several undergraduate play the roles of travelers. Finally, we will test the system in some small trips by foot, bus or car around Santa Cruz, Palo Alto and Redwood City.

\section{Conclusions and Future work}

In this paper we describe the plan and progress of our research on building SCATEAgent, an agent-based context-aware ATIS environment for multi-modal travel. The autonomic, collaborative, and intelligent nature of software agents helps to bring the very much desired and needed dynamic, flexible, and highly-personalized character to current ATIS systems, which are relatively simple and isolated. One key issue is to build consistent ontologies over different agents and external services and models amiable to manipulation through rule-based systems, state machines, machine learning tools, or other intelligent elements.

A challenge is how much intelligence is needed to get a useful amount of benefit from adaptive, flexible goal-directed processing [VanHilst and Griss, 2003]. A key strategy is to initially avoid very deep AI and complex natural language understanding, and look for less intelligent agents. Currently we are combining rule-based techniques (JESS) with our state machine extensions to JADE Griss et. al 2003] to make it easier to handle complex negotiation protocols. Currently, the state machines manage the protocols used in the negotiation between agents, such as agreeing on a common time and location for several travelers to meet. Other negotiations involve (re-)checking with the traveler should the priorities, plans, or schedules change. We are investigating mechanisms to further integrate a combination of rule-based, machine learning, information retrieval and data mining techniques into a coherent "intelligence" toolkit, consistent with the models described above, and convenient for use inside the JADE agents. Our overall approach is to structure knowledge and rules within each agent to support a simplified form of goal-directed computation. Goals are decomposed into smaller goals and assigned to specific components in the system. Each system component agent performs behaviors in pursuit of its goals. Large systems with complex behavior are formed by collections of narrowly focused agents, each acting in its own self-interest.

Another major challenge is to develop a uniform agent human interface. Since the traveler is always on the move and will encounter different devices and/or different interaction modalities (speech, key-stroke, stylus, etc) during the trip, we need to develop a framework that helps human agent interface to roam smoothly and seamlessly from one device to another, one modality to another. We have designed such a framework where dialogue agent, device agent, proxy agent, and other utility agents collaborate to maintain a continuous dialogue and manage or trim the dialogue according to the interaction context, task, and user preference.

Finally, we plan to scale the system to a larger pilot test, used by some of the researchers on a regular basis.

\section{Acknowledgements}

This work was produced with research grant no. NSF CMS-0339251 SC 20030921 funded by NSF and the Department of Transportation Federal Highway Administration, and with support from HP Laboratories. We also appreciate the contributions of Ryan Delucchi. 


\section{References}

[Adler 1998] Adler, J. \& Blue, V., Toward the design of Intelligent Traveler Information Systems, cooperative multi-agent transportation management and route guidance system, Transportation Research, 6C(3), June 1998, 157 172.

[Adler 2002] Adler, J. \& Blue, V. A cooperative multi-agent transportation management and route guidance system, Transportation Research, 10C(5-6), Oct-Dec 2002, 433454.

[Anderson \& Rnnbom 1999] Andersson, L. and Rnnbom, A. Intelligent Agents - A New Technology for Future Distributed Sensor System? Informatics 1999. http://citeseer.nj.nec.com/andersson99intelligent.html

[Appiani 1999] Appiani, E., Martelli, Maurizio, and Mascardi, Viviana, "A Multi-Agent Approach to Vehicle Monitoring in Motorway”, Technical Report DISI TR-0013, 2000,

http://www.disi.unige.it/research/Logic_programming/agen t_pubs.html

[Bauer, et al, 2001] Bauer, B. Müller, P., Odell, J. Agent UML: A Formalism for Specifying Multi-agent Interaction in Agent-Oriented Software Engineering, Paolo Ciancarini and Michael Wooldridge eds., Springer, Berlin, pp. 91-103, $2001 . \quad$ (See also http://www.auml.org/auml/supplements/main.shtml)

[Blechschmitt \& Strodecke, 2002] Blechschmitt, E \& Strodecke, C., An architecture to provide adaptive, synchronized and multimodal human computer interaction, Proceedings of the Tenth ACM International Conference on Multimedia, Juan-les-Pins, France, December, 2002

[BlueJADE] http://www.bluejade.net/public_resources/director/

[Burmeister et al, 1997] B. Burmeister, A. Haddadi, and G. Matylis. Application of multi-agent systems in traffic and transportation. In IEE Proceedings of Software Engineering 1997 pages 51--60.

[Cellphone] Facts about cellphone use http://www.insweb.com/learningcenter/specialreports/cellphones/facts.htm

[Chen, et. al, 2000] Chen, Q., Hsu, M., Dayal, U., Griss, M.: Multi-Agent Cooperation, Dynamic Workflow and XML for E-Commerce Automation, Autonomous Agents 2000, June (2000), Barcelona, 255-256.

[CityTraffic] CityTraffic - An integrated system for traffic planning, management and information in high-density urban areas http://www.citytraffic.de/ct_eng.html See also http://www.ercim.org/publication/Ercim_News/enw46/mue hlen.html

[CoolTown] CoolTown - HP Laboratories-developed, open source technology for context-aware web-based applications. http://www.cooltown.hp.com/cooltownhome/index.asp

[Cowan \& Griss, 2002] Cowan, D. and Griss, M., Making Software Agent Technology Available to Enterprise Applications, 1st International Workshop on "Challenges in Open Agent Systems", AAMAS'02, Bologna, Italy, July 2002

[Das 1999] Das, S., Bowles, BA. Houghland, CR., Hunn, SJ., Zhang, Y. A knowledge based model of traffic behavior in freeways, Proceedings of the 1999 ACM Symposium on
Applied Computing, , San Antonio, Texas, United States, 14-18.

[Erol 1999] Erol, K., Levy, R., \& Wentworth, J., Application of Agent Technology to Traffic Simulation, Federal Highway http://www.tfhrc.gov/advanc/agent.htm - 1999

[Fernandes \& Oliverira 1999] Fernandes, JM. \& Oliveira, E., "TraMas: Traffic Control through Behaviour-based MultiAgent System", Proceedings of the Fourth International Conference on The Practical Application of Intelligent Agents and Multi-Agent Technology (PAAM99),pp 457458, London, April $1999 . \quad$ See http://www.ieeta.pt/ jfernan/tramas/

[FreeWay2000] http://www.freeway2000.com/ provides a phone number (for LA: (213) 614-2000), and a rather tedious and laborious VoiceXML-like interface.

[Goni, et al, 2001] Goni, A., A. Illarramendi, A., E. Mena, E., Y. Villate, Y., \& J. Rodriguez, J. "Antarctica: A multiagent system for internet data services in a wireless computing framework". In NSF Workshop on an Infrastructure for Mobile and Wireless Systems Scottsdale, Arizona (USA), October 2001.

[Grimnes, et al.] Grimnes, G. A. A., Charlmers, S., Edwards, P., Preece, A., "GraniteNights - A Multi-Agent Visit Scheduler Utilizing Semantic Web Technology”, http://www.csd.abdn.ac.uk/ ggrimnes/pubs/GraniteNights. pdf

[Griss \& Kessler, 2002] Griss, M. and Kessler, RR. "Achieving the Promise of Reuse with Agent Components.” First International Workshop on Software Engineering for Large-Scale Multi- Agent Systems, ICSE, May 2002, p. 1-9.

[Griss 2000] Griss, M.: My Agent Will Call Your Agent, But Will It Respond, Software Development Magazine, Feb, 2000 .

[Griss et al., 2002] Griss, M., Letsinger, R., Cowan, D. Sayers, C., VanHilst, M., Kessler, R.: CoolAgent: Intelligent Digital Assistants for Mobile Professionals - Phase 1 Retrospecitve. HP Laboratories technical report HPL-200255(R), July 2002.

[Griss et. al, 2003]Griss, M., Fonseca,S., Cowan, D., and Kessler, R., Using UML State Machines Models for More Precise and Flexible JADE Agent Behaviors, AAMAS AOSE workshop, Bologna, Italy, July 2002.

[Guinchiglia, et. al., 2003] Guinchiglia, F., Myopoulos, J. and Perini, A. The Tropos Software Development Methodology: Process, Models and Diagrams. AgentOriented Software Engineering III, Bologna, July 2002. Springer LNCS 2585. pp 162-173.

[Hidas 2002] Hidas, P., Vehicle Interactions by Intelligent Agents, University of New South Wales, June 2002. http://www.civeng.unsw.edu.au/staff/hidas.p/sitras.htm

[IAI] Intelligent Automation, Inc., agent-based traffic simulator using OpenCybele and AASIM. http://www.i-ai.com

[Iteris] Iteris offers real time maps, has a concept of Personalized Traveler Information, is one of two companies selected to develop a national ITS architecture. http://www.iteris.com/. . See real time maps, http://traffic.iteris.com/html/internal/frameset/sfo.html 
[Jade] Bellifemine, F., Poggi, A., Rimassi, G.: JADE: A FIPA-Compliant agent framework, Proc. Practical Applications of Intelligent Agents and Multi-Agents, April (1999), 97-108; also http://sharon.cselt.it/projects/jade

[Jess] Jess ${ }^{\mathrm{TM}}$ A forward and backward chaining rule system, written in JAVA. http://herzberg.ca.sandia.gov/jess/

[Jini] Jini Network technology http://wwws.sun.com/software/jini/

[Katwijk 2002] van Katwijk, R., and van Konigbruggen, P., Coordination of traffic management instruments using agent technology, Transportation Research Part C 10 (2002) 455-471, Elsevier.

[Kendall, 1999] Kendall, EA."Role Model Designs and Implementations with Aspect-oriented Programming," in Proc. of OOPSLA 99, Oct, Denver, Co., ACM SIGPLAN, 353- 369.

[Kindberg 2002] Kindberg, T., et al., People, Places, Things: Web Presence for the Real World, HP Labs tech report, HPL-2001-279, 2001,. In proceedings WMCSA2000. ACM MONET (Mobile Networks \& Applications) Vol. 7, No. 5 (October 2002).

[Lapin 2000] Lapin, J., Advanced Traveller Information Service(ATiS): Who are ATIS customers, US DOT, Jan 2000, \#12885

[Lapin 2002a] Lapin, J., Advanced Traveller Information Service(ATiS): What do ATIS Customers Want, US DOT, Jan 2000, \#12884

[MobilityTechnologies] MobilityTechnologies owns traffic.com (used to be traffic.com) http://www.traffic.com - provide real-time traffic information via TrafficPulse, digital sensors, real-time updates on web, email, soon wireless. Provide an annotated map, shows incidents, etc.

[NextBus] Nextbus - GPS enabled busses in San Francisco http://www.nextbus.com

[NITSA] US Department of Transport, National ITS Architecture, http://www.its.dot.gov/arch/arch.htm

[Odell 2001] Odell, J., Van Dyke Parunak, H., Bauer, B. Representing Agent Interaction Protocols in UML, in Agent-Oriented Software Engineering, Paolo Ciancarini and Michael Wooldridge eds., Springer, Berlin, pp. 121140, 2001.

[PeMS] Freeway Performance Measurement Project Web Site, funded by PATH http://pems.eecs.berkeley.edu/login.phtml - $\quad$ collects historical and real-time data flow, load and incident from Freeways, and presents it in low-cost, easy-to-use forms, on the web or via FTP.

[Roozemond 1996] Roozemond, D.A., Intelligent traffic management and urban traffic control based on autonomous objects; in Proceedings of the sixth Artificial intelligence, simulation, and planning in high autonomy systems international conference), IEEE, 1996. (ftp://cci.ct.tudelft.nl/DR_AISP.pdf)

[Rosen 1970] Rosen, DA., Mammano, FJ., \& Favort, R. An Electronic Route Guidance System for Highway Vehicles, IEEE Transactions on Vehicular Technology, VT-19, 1970.

[SFBayTraffic] A personalized alerts service can relay important traffic information to you on your cellphone, pager (and any device that can receive email) when you are on the road, or about to get on it, using a profile. http://www.sfbaytraffic.com

[Shoham 1993] Shoham, Y., "Agent-Oriented Programming," Artificial Intelligence, Vol. 60, No. 1, 1993, Pp. 139-159.

[Sutton \& Osterweil, 1997] Sutton, SS. And Osterweil, LJ. "The design of a next generation process programming language," Proceedings of ESAC-6 and FSE-5, Springer Verlag, 1997, pp. 142-158.

[Telcontar] TelContar http://www.telcontar.com/ is a "traffic manager product, written in Java, uses Teleatlas SmartRoute and TrafficCast.

[TeleNav] A Nextel service that uses GPS enabled cell-phones to provide-turn-by-turn navigation information in a cell-phone. Is voice enabled, does automatic reroute updates http://www.telenav.net/telenavnextel

[TrafficDodger] Astartup that provides a MapQuest like interface, over TeleAtlas data, with some incident, event and time travel annotations. http://www.trafficdodger.com/ . Will annotate best route, give estimated travel time, etc.

[VanHilst and Griss, 2003] VanHilst, M. \& Griss, M. GoalDirected Design for Proactive and Intelligent Device Collaboration., KES 2003: 841-848.

[ViaMoto] Motorola's location-ware and traffic services. http://www.motorola.com/mobileinternet/

[Ward et al., 2002] Ward, A., Terman, C., Saif, U.: Goal Oriented System Semantics, March 2002 http://cag.www.lcs.mit.edu/ umar/publications/Goals_abst ract.pdf

[WestwoodOne] Westwood One (Metro Networks, SmartRoute). "SmartRoute Systems provides the very latest in local traffic, news, sports and weather information to wireless, Internet, in-vehicle navigation systems and voice portal customers, in multiple formatshttp://www.metronetworks.com/

[Wojcik 2000] Wojcik, LA., Campbell, K.C., Cooper W.W., \& Greenbaum, DP, "Modeling Distributed Human Decision-Making in Traffic Flow Manageent Operations”, Mitre, June 2000. Describes CAASD agent-simulator for advanced air traffic management, in a complex, adaptive environment.

[Wooldridge et al. 2000] Wooldridge, M., Jennings, NR., and Kinney, D., "The Gaia methodology for agent-oriented analysis and design,” Autonomous Agents and MultiAgent Systems 3, Kluwer, 2000, pp. 285-312.

[Zeus] Nwana, H., Nduma, D., Lee, L., Collis, J.: ZEUS: a toolkit for building distributed multi-agent systems, in Artificial Intelligence Journal, Vol. 13, No. 1, (1999) 129186; also http://more.btexact.com/projects/agents/ZEUS 